\title{
Lapurdum
}

Euskal ikerketen aldizkaria | Revue d'études basques |

Revista de estudios vascos | Basque studies review

$8 \mid 2003$

Numéro VIII

\section{L'évolution des frontières de l'état français et de l'identité culturelle basque : perspectives anthropologiques}

\section{David Seth Murray}

\section{OpenEdition}

\section{Journals}

Édition électronique

URL : http://journals.openedition.org/lapurdum/1164

DOI : 10.4000/lapurdum. 1164

ISSN : 1965-0655

Éditeur

IKER

Édition imprimée

Date de publication : 1 novembre 2003

Pagination : 375-388

ISBN : 9782867813436

ISSN : $1273-3830$

Référence électronique

David Seth Murray, «L'évolution des frontières de l'état français et de l'identité culturelle basque perspectives anthropologiques », Lapurdum [En ligne], 8 | 2003, mis en ligne le 01 mai 2009, consulté le 01 mai 2019. URL : http://journals.openedition.org/lapurdum/1164; DOI : 10.4000/lapurdum.1164 


\section{Seth Murray}

\section{Lévolution des frontières de l'état français et de l'identité culturelle basque: perspectives anthropologiques}

\section{Introduction}

Le Pays Basque se situe à la périphérie géographique et culturelle de la France, et nous offre un scénario où divergences entre identités nationales et locales accentuent le caractère hétérogène de l'état français. Cette problématique peut être éclaircie en cadrant l'identité culturelle basque en tant que symptôme de tensions historiques entre le centre et sa périphérie qui se manifestent en zones frontalières. Nous suggérons donc trois objectifs dans ce travail: premièrement, nous interrogerons la pertinence de l'idée de frontière dans le cadre de l'état national pour les sciences sociales. Deuxièmement, nous porterons un regard anthropologique sur le rôle de la frontière dans la construction des identités diverses, vue à travers l'exemple des rapports historiques entre l'état français et le Pays Basque. Et en dernier lieu, nous analyserons l'influence historique de la frontière et de l'état français dans une commune pastorale basque.

Une frontière est conçue tout d'abord comme un lieu où états se rencontrent. Mais les rapports étatiques que constitue une frontière interpellent aussi les communautés locales ci-situées, celles-ci pouvant être à l'origine d'une dynamique socio-politique surpassant leurs positions marginales. En effet, le Pays Basque apparaît comme un lieu d'étude privilégié puisqu'il est à la fois situé à la frontière géopolitique entre la France et l'Espagne, tout en étant simultanément un lieu de contact culturel.

À cause de la situation géographique du Pays Basque au sein de la France, la communauté basque a eu affaire à l'état depuis des siècles. Mais 
ce n'est pas la présence de l'état français en elle-même qui détermine le fonds ou la forme d'une identité basque, malgré le fait que celle-ci est historiquement façonnée par la présence inéluctable de la France. L'identité culturelle basque est structurellement liée à la France puisque les Basques sont contraints de configurer leurs identités culturelles avec l'état français comme arrière-plan. La construction d'une identité culturelle résulte de processus dialectiques qui émanent de la conjoncture de multiples éléments sociaux pouvant être manifestés à des échelles à la fois locales et nationales.

Pour examiner les rapports historiques entre communautés basques et l'état français, nous interrogerons la manière dont le pastoralisme basque s'est progressivement adapté aux contraintes qui lui ont été imposées par l'état national dans ce contexte frontalier. Les pratiques d'utilisation des terres communales par les bergers de la vallée de Baïgorry (département des Pyrénées-Atlantiques) sont soumises depuis plusieurs siècles à des contraintes imposées par l'état suite à des traités établis entre la France et l'Espagne. Ces traités régissent les pratiques pastorales autour de la frontière, et ont eu par conséquent une influence sur la construction de l'identité culturelle basque dans cette région. Nous voulons penser que l'analyse anthropologique présentée ici nous permettra de juxtaposer l'évolution d'une frontière de la France et de l'identité culturelle basque.

\section{Frontières : à la périphérie et au centre de l'état}

Une frontière est fréquemment conçue comme un simple endroit où des limites étatiques se rencontrent. Il est vrai qu'une frontière est un lieu de rencontre internationale, mais celle-ci interpelle également des structures sociales autres que les institutions de l'état: une frontière influence aussi les communautés locales s'y trouvant, les obligeant à y faire face. En revanche, cela ne signifie pas que ces communautés sont passives face à une diplomatie internationale, puisqu'une population frontalière peut prétendre à une influence socio-politique qui aille bien au-delà de sa marginalité géographique. La frontière de l'état français située au Pays Basque est géopolitique dans le sens qu'elle se situe aux limites des états souverains de la France et de l'Espagne. Mais ce lieu est également une zone de contact culturel entre Basques, Français, et Espagnols (Douglass 1977). Il est donc important de comprendre que cette frontière pyrénéenne est autant le résultat de processus géopolitiques que la conséquence d'une construction culturelle. 
Nous estimons que les régions frontalières peuvent s'avérer être des terrains de recherche remarquables lors d'études analysant la structure sociale de l'état, puisqu'une frontière est constamment assujettie à une contestation et à une (ré) interprétation des groupes ayant des intérêts divers. Les frontières sont génératrices non seulement de rapports entre les acteurs sociaux de la périphérie et du centre de l'état national, mais les frontières produisent également un effet sur les rapports entre les divers groupes situés en marge de l'état (Donnan et Wilson 1994). Nous suggérons ici que les chercheurs en sciences sociales n'ont pas suffisamment porté leur regard vers l'état national et son impact sur les pratiques de la vie quotidienne dans ces communes frontalières, et nous concluons que cette insuffisance nous détourne d'une meilleure connaissance des manières dont la souveraineté nationale est vécue au niveau de l'individu. Donnan et Wilson estiment que, « trop souvent, les constructions culturelles qui symbolisent les limites avec d'autres communautés et avec d'autres nations, sont diluées par le gros plan qu'occupent les rapports nationaux et internationaux. Les cultures frontalières illustrent les rapports dialectiques qui existent entre divers groupes sociaux, ainsi que les rapports entre ceux-ci et d'autres niveaux de pouvoir socio-politique, et surtout avec celui de l'état » (p. 10)!

Nous soulignons ici que l'état doit être compris dans son contexte culturel, ce qui de façon générale devrait inclure un système d'idées, de signes, de mythes, et de valeurs, etc. qui sont partagés par une population (Gellner 1983). De plus, Benedict Anderson affirme que l'état national se résume à une « communauté imaginaire » par le fait que les citoyens d'une nation peuvent « reconnaître » les autres individus membres de cette même nation, même s'ils ne se connaissent ni côtoient pas personellement (Anderson 1983). Selon cette logique, par exemple, un Irlandais, un Japonais, ou un Costaricain (ce ne sont après tout que des exemples types) est supposé pouvoir reconnaître un autre Irlandais,... quand bien même ils ne se seraient jamais rencontrés auparavant. C'est donc par l'intermédiaire de ce système de symboles mutuellement partagé et soutenu que citoyens d'un même état sont supposés être capables de s'entre-reconnaître. De ce fait, la cohésion sociale au sein d'un pays résulterait de la loyauté et de la solidarité des individus envers le concept de l'état national.

Il s'ensuit donc que l'état national est une entité complexe et variée, dépendant autant de l'adhésion mentale des individus que de réalités empi-

Toutes les citations du texte sont traduites de l'anglais par nous. 
riques. La construction socio-culturelle d'une nation est par extension un processus qui dépend de la culture et de la conscience, du sentiment et de la perception, et finalement, de la capacité d'une nation à consolider sa légitimité, son autorité, et sa souveraineté. Cet argument est encore plus valable lorsque nous analysons la construction socio-cuturelle de l'état national dans ses régions frontalières. Mais il ne faut pas en déduire que la construction culturelle d'un État national est uniquement un phénomène qui émane du centre de pouvoir politique, puisqu'une identité et une conscience nationale se construisent également avec la participation d'individus " ordinaires »; c'est-àdire des personnes qui ont une influence et un pouvoir marginal par rapport aux institutions de l'état. Ces gens « ordinaires » participent tout de même à la définition culturelle de leur communauté locale et à celle de leur propre identité culturelle, souvent en se contre-balancant à l'identité dictée par l'état national. Michael Wintle décrit ce processus comme « une invention de tradition » ce qui serait une situation résultant d'une part de l'imposition d'un modèle d'unité nationale et d'autre part, de la perception et du vécu de ce modèle par une communauté locale (1996). Cette tension binaire souligne pour nous la multiplicité de manières dont l'état national peut être perçu, transformé, et vécu par des individus et des groupes différents au niveau local, et non seulement à l'échelle nationale. Par conséquence, « une identité [culturelle] ne peut être construite uniquement par le centre. Il est également nécessaire d'avoir des initiatives locales et décentralisées afin d'agréer ou de défier les actes du gouvernement. Les militants locaux, les groupes ethniques ou minoritaires, etc. sont tous engagés dans un processus de construction identitaire qui se place au sein d'une identité [régionale ou nationale] plus vaste » (Wintle 1996 : p. 21).

Il est donc évidemment difficile de parler d'une identité nationale sans faire référence aux défis intrinsèques qui sont souvent vocalisés par le discours et les pratiques des zones frontalières. Le Pays Basque nous offre précisément un exemple où les divergences de l'identité nationale soulignent une hétérogénéité culturelle au sein même de l'état national, et par extension, ces contradictions remettent en cause le succès du projet révolutionnaire français. Suivant l'analyse de Jurgën Habermas, si l'état national se conçoit en tant que communauté unifiée et possédant une identité nationale fiable, c'est parce que l'état se pourvoit des symboles et des institutions qui renforcent l'affiliation et l'affinité nationale (1992). La manière dont certains individus et groupes des régions frontalières de la France interagissent avec l'état remet en cause l'hégémonie que l'état souhaiterait maintenir par le biais de l'image d'une France homogène; c'est en créant et projetant l'image d'un paysage culturel et politique uniforme que l'état français serait vraisemblablement 
vécu de façon identique par tous ses citoyens.

L'argument précédent évoque pour nous une thèse parallèle située dans l'œuvre d'Eugen Weber (1983). En bref, Weber affirme que la consolidation sociale nationale est provoquée au centre de l'état par son élite socio-politique, et qu'elle s'en propage progressivement vers les régions périphériques par l'intermédiaire des institutions telles que l'éducation nationale ou le service militaire. En d'autres mots, c'est l'état qui crée, définit, et répand une identité nationale qui à son tour façonne le citoyen rural - voire marginal - à l'image du citoyen français idéal. L'argument de Weber sous-entendrait bien sûr que le citoyen marginal ne peut exercer qu'une influence restreinte sur la construction de son identité culturelle, et qu'en fin de compte, l'individu n'aurait qu'un contrôle très limité de son choix d'appartenance identitaire. En revanche, l'historien Peter Sahlins nous propose une interprétation alternative de l'identité culturelle qui suggère que la construction historique de l'état résulte en grande partie des évènements sociaux qui se déroulent aux marges du territoire national plutôt qu'au centre (1989). Ce serait donc autour de ces pôles limitrophes de l'état que se rencontrent et se côtoient forces locales et internationales, et c'est de ces rencontres que les institutions étatiques puisent leurs motivations pour une identité collective et nationale. Selon cette interprétation, c'est la « frontière, plus que le centre, qui apparaît comme le lieu privilégié de l'existence de la nation" (Gemie 1998 : p. 313). Nous tenterons de fournir dans ce travail un complément aux recherches de Peter Sahlins en Cerdagne avec des données historiques provenant d'une autre région frontalière de la France: le Pays Basque.

\section{Frontière et identité culturelle : perspectives anthropologiques}

Nous définissons l'identité culturelle dans cet article comme la représentation sociale du soi; c'est-à-dire que l'identité culturelle se construit à partir de divers critères idéologiques dans lesquels l'être humain puise pour se définir dans son monde social. La notion d'identité ne doit pas être considérée en tant qu'une catégorie évidente, puisqu'il faut la nuancer par ses pratiques, son actualisation, même par sa cognition. C'est pourquoi l'identité culturelle élucide également les allégeances qu'une personne ou qu'un groupe peut avoir envers un lieu, un passé, une langue, ou une pratique. Mais l'identité culturelle n'est pas influencée et ne provient pas d'une seule source d'allégeance, mais résulte plutôt d'une combinaison de plusieurs de celles-ci. 
L'individu possède plusieurs identités coexistantes de ces sources d'allégeances culturelles, et c'est en fonction de l'environnement social que la personne se représente - ou est perçue par autrui - d'une certaine manière. Ces éléments deviennent des "marqueurs identitaires " qui sont ensuite appropriés ou attribués comme étant des traits caractéristiques de personnes associés à une identité culturelle spécifiques. En d'autres mots, ces marqueurs peuvent très bien représenter des stéreotypes culturels.

Il nous semble qu'une région frontalière comme le Pays Basque est un lieu propice pour étudier la formation et l'expression de l'identité culturelle (Jenkins 2000). L'anthropologie est une discipline qui porte son regard analytique le plus souvent sur des communautés humaines à l'échelle locale, et de ce fait, il est surprenant que les anthropologues n'aient pas plus régulièrement examiné le rôle socio-culturel des frontières au sein de ces communautés. Ceci est d'autant plus remarquable puisque nous suggèrons ici qu'une anthropologie des frontières pourrait nous aider à comprendre la manifestation de l'identité culturelle aux limites géopolitiques de l'état. C'est en analysant la manière dont les habitants de ces régions vivent la frontière à travers leurs vies quotidiennes, autant par nécessité que par obligation, que l'anthropologie se retrouve capable d'approfondir nos connaissances sur le présent et l'avenir des enjeux sociaux et nationaux, et c'est ainsi que l'anthropologie pourra offrir une contribution considérable aux sciences sociales.

Un certain nombre d'études intéressantes ont été menées par des anthropologues au cours de la deuxième moitié du vingtième siècle; notamment celle de Frederik Barth à propos des coutumes socio-économiques de groupes ethniques différents dans le sous-continent Indien (1969). Mais nous notons également l'analyse comparative de deux communes frontalières du Tyrol faite par John Cole et Erik Wolf (1974), ainsi que les recherches de William Douglass sur l'influence de la frontière dans des communes basques de la Navarre $(1975,1977)$. Mais ces études anthropologiques qui abordent toutes d'une façon ou d'une autre la notion de frontière demeurent défaillantes dans leurs fonds, soit puisqu'elles s'étendent trop sur une prétendue homogénéité des groupes en décalage avec la réalité culturelle des frontières étatiques, soit parce que ces études établissent des traitements monographiques de communautés qui accentuent des nuances sociales minutieuses au détriment de l'impact culturel à long terme de l'état. En revanche, ces études ont eu un apport théorique important pour l'anthropologie socio-culturelle nord-américaine, y compris pour les études archéologiques qui interrogaient le dynamisme du rôle social des frontières dans l'expansion des états proto-historiques (Brumfiel et Fox 1994; Gibson et 
Gesolowitz 1988; Renfrew et Cherry 1986). Mais malgré ces apports, nous estimons que l'anthropologie demeure jusqu'à présent bien en deça de son potentiel puisqu'elle n'a pas su utiliser ses connaissances du nationalisme et de l'ethnicité pour en extraire une contribution considérable pour les sciences sociales.

Dans une région frontalière comme celle du Pays Basque, il est particulièrement important de considérer l'évolution des rapports entre les échelles nationale et locale, et d'analyser quelles ont été ses influences sur la construction de l'identité culturelle sur la longue durée (Jenkins 2000). Nous suggérons que l'état a depuis fort longtemps tenté d'intégrer le Pays Basque au sein de d'une nation française unifiée, et que ces efforts n'ont pas toujours abouti à l'assimilation identitaire des Basques. Donc l'identité culturelle basque serait plûtot « un résultat des processus de négociations entre le centre et sa périphérie, et une identité n'est pas simplement imposée par le centre parisien » (Lammers 1999 : p. 82). À l'échelle locale, une identité culturelle basque n'a pas été supplantée ou remplacée par l'enveloppe d'une identité française, préconisée par l'état au travers de ses institutions. Au contraire, le résultat de ces négociations entre centre et périphérie est une convergence de multiples identités culturelles, à la fois basque et française. Même si ceci ne remet pas en cause l'intégralité territoriale de la nation, l'état demeure incapable de forger et de consolider une identité nationale unique, et cela en dépit des siècles de tentatives au Pays Basque tout comme dans d'autres régions de la France.

\section{Identité culturelle, frontière, et terres communales}

Une étude portant son regard analytique sur des terres communales doit interroger la situation locale sans négliger de superposer des éléments diachroniques et extra-locaux. Il existe certains atouts dans une étude des terres communales qui relèvent du fait que cet espace est un reflet matériel de sa gestion par les hommes. C'est ainsi que des recherches sur les terres communales peuvent servir de point de départ pour mieux comprendre quelles sont les causes et les conséquences des changements socio-économiques dans une région. Certes le vécu historique des Basques au sein de l'état français occasionne un impact sur la construction de leur identité collective, mais ces expériences doivent également se retrouver dans leurs practiques au quotidien (Greenwood 1976). Nous pensons que la gestion de terres communales peut indiquer certaines facettes de l'identité culturelle basque puisque la ges- 
tion locale de ces terres a connu l'impostion de certaines contraintes par l'état français. Pour servir d'illustration, nous présentons à présent les résultats de nos recherches en cours dans la vallée de Baiggorry, située dans les Pyrénées à proximité de la frontière entre la France et l'Espagne.

Déjà lors du Moyen Âge, les communautés basques de la vallée de Baïgorry coopéraient entre elles poour réglementer la gestion des terres communales. Des représentants des familles de villages se réunissaient régulièrement lors de Cours Générales pour établir les paramètres pour gérer la vie sociale, les obligations et devoirs de familles individuelles, ainsi que les rapports entre villageois et les représentants du gouvernement ou de la nobilité (Cavaillès 1910). Entre autre, ces assemblées déterminaient également les règles qui gouvernaient la gestion et l'utilisation des terres communales (Etchelecou 1991). À cette époque, une grande partie de la vallée de Baïgorry était désignée comme terre communale (ou país indiviso dans les documents espagnols originaux), et par conséquent, pendant des siècles les habitants ne pouvaient ni occuper ni exploiter ces terres de façon continue. Dès le début du treizième siècle, lorsque le royaume de Navarre avait le contrôle de la vallée de Baïgorry, il était interdit d'y bâtir des habitations et de plus, la plupart des droits usufruits étaient réservés pour les plaisirs de la cour royale (Gautier Dalché 1992). Nous pourrions interpréter ce décret comme un des premiers enregistrements de restrictions vis-à-vis de l'exploitation des terres dans cette région. Donc cette situation semblerait sérieusement limiter les possibilités usufruitières des habitants locaux de la vallée de Baïgorry, ou du moins semblerait les avoir circonscrits à une utilisation saisonale.

Le royaume de Castille et Aragon annexa le royaume de Navarre en 1512, ainsi que toutes ses possessions, ce qui incluait la vallée de Baïgorry dans les Pyrénées. L'Espagne, avec le consentement de la monarchie française, a préféré maintenir la vallée de Baïgorry comme terre communale afin de préserver un butoir entre les deux états. Il semblerait que jusqu'au milieu du seizième siècle, plus de la moitié de la vallée de Baïgorry a été exploitée en tant que terre communale (Etchelecou 1991). Mais en dépit des lois qui régissaient la vallée à cette époque, il y a avait un nombre important d'agriculteurs basques qui y étaient clandestinement installés. Ces agriculteurs basques, pour la quasi-totalité bergers, venaient soit des villages de Saint-Étienne-deBaïgorry au nord, soit du val d'Erro au sud (Cavaillès 1910). Les représentants de l'état français ont tenté de restreindre le peuplement de la vallée de Baïgorry en y démolissant régulièrement les fermes et autres bâtiments agricoles (Goyheneche 1975). 
L'utilisation et la gestion locale des terres communales soulignent les tensions croissantes entre la périphérie basque et l'état français en vue des restrictions imposées. De plus, une compétition locale commençait entre différents villages basques des deux versants des Pyrénées qui souhaitaient avoir accès aux pâturages dans la valiée de Baïgorry; ce qui ne fait que compliquer davantage les rapports entre la France et l'Espagne. Les rivalités entre villages étaient liées au fait que les terres communales représentaient un espace qui pouvait offrir aux exploitations pastorales un puissant atout économique. La convoitise pour la vallée de Baïgorry était d'autant plus importante que la croissance démographique du début du dix-huitième siècle avait obligé de nombreux habitants de Saint-Étienne-de-Baïgorry qui n'avaient pas de terre à s'établir dans la vallée (Viers 1950). Ce flux d'habitants de Saint-Étiennede-Baïgorry a conduit à la création de nouveaux villages dans la vallée, ce qui favorisa pour eux l'utilisation des terres communales au détriment des bergers du val d'Erro par example. Donc, en plus des restrictions sur l'habitation dans la vallée de Baïgorry qui mettaient ces nouvelles communautés dans une position illégale vis-à-vis de l'état, viennent s'ajouter les disputes locales entre les communautés basques d'Erro et de Saint-Étienne-de-Baïgorry.

Ces tensions locales connaitront en fin de compte des incidents violents, comme par exemple en 1768 lorsque plusieurs douzaines d'habitants du val d'Erro sont grièvement blessés par un groupe de bergers venu de SaintÉtienne-de-Baïgorry (Lefebvre 1933). L'occupation et l'utilisation des terres communales de la vallée de Baïgorry seront l'objet d'intenses négociations entre la France et l'Espagne, qui aboutiront en 1785 avec le traité d'Elizondo. En bref, cet accord inter-gouvernemental établit la « ligne d'Ornano » qui divisait la vallée de Baïgorry en deux zones pastorales distinctes. Le traité d'Elizondo stipulait que la partie nord de la vallée était réservée aux bergers de Baïgorry, tandis que les habitants du val d'Erro avaient le privilège usufruitier de la partie sud, ce qui laisserait croire que la polémique de l'utilisation des terres communales était résolue. Ce traité a également enfin établi la légalité des nouveaux villages dans la vallée, désigné par ailleurs dans une carte qui accompagnait ce document comme país indivisos (pays indivis).

Le traité d'Elizondo est important par le fait que les états français et espagnol ont collaboré ensemble pour résoudre le dilemme du statut de la vallée de Baïgorry, ce qui demeurait dans son fond un problème local. Nous suggérons que ceci représente pour une des premières fois un exemple de coopération étatique qui par extension, a souligné la position marginale et subordonnée des communautés basques par rapport à des décisions politiques nationales. Néanmois, ce traité n'a pas décisivement résolu les rivalités et tensions entre les communautés basque de cette zone frontalière. 
En effet, suite au traité de 1785 , les disputes se sont renouvelées entre les habitants de Saint-Étienne-de-Baïgorry et du val d'Erro (García-Ruiz et Lasanta-Marínex 1993). Les tensions étaient encore dûes à des contestations sur l'utilisation des resources d'eau et de pacage dans la vallée de Baïgorry (Cavaillès 1931a). Ce n'est qu'en 1856 qu'un nouvel accord conciliatoire fut établi, le traité des Pyrénées. Celui-ci établit la « ligne d'Ornano » du traité de 1785 comme la frontière définitive entre la France et l'Espagne dans la vallée de Baigorry (Chaussier 1989). Le traité de 1856 cèda l'utilisation des pâturages buttant des deux côtés de la frontière dans la vallée aux bergers de Baïgorry et leur a permis d'y construire leurs demeures, tandis que les habitants d'Erro n'avaient que le droit d'y faire pacager leurs troupeaux pendant les estives, sans avoir le droit d'y habiter (Cavaillès 1931b). Cette entente favorisait bien évidemment les Basques habitant en France au détriment de leurs homologues habitant du coté espagnol, mais l'état français a consenti lors du traité des Pyrénées à verser un paiement annuel, un versement qui continue encore jusqu'à nos jours (Laborde 1983).

Une conséquence du traité de 1856 est que la zone située jusqu'à une distance de cinq kilomètres au sud de la frontière politique est reconnue comme territoire espagnol, mais son utilisation est réservée principalement aux habitants du territoire français. C'est au sein de ce contexte international que les agriculteurs basques habitant autour de cette frontière géopolitique et culturelle ont commencé à forger leurs identités culturelles par rapport aux intérêts de l'état français.

\section{Conclusion}

En somme, le rôle des terres communales et l'évolution de la frontière dans cet exemple révèlent des négociations historiques entre une région périphérique et l'état français. Les Basques ont subi les conséquences de décisions exogènes mais sans renoncer à subvenir à leurs besoins économiques, et c'est cette dialectique qui sert comme un tour de potier à l'identité culturelle basque. Durant les dix-neuvième et vingtième siècles, les diversités régionales ont été des phénomènes complexes pour l'état français. Afin que la France solidifie son hégémonie, politique sur son territoire, elle a établi des lois qui liaient ses régions au centre. La révolution française et l'idéologie jacobine étaient des forces homogénéisantes qui tentaient de forger une prétendue unité nationale, même dans des régions comme le Pays Basque où celles-ci avaient peu de pertinence dans la vie quotidienne des gens. Après des 
siècles de tentatives de consolidation politique, une réelle diversité culturelle persiste encore sur le territoire de la France, et surtout dans ses régions frontalières.

Nous estimons qu'il serait naïf de tenter de comprendre la situation historique et contemporaine du Pays Basque et de l'identité culturelle sans la placer au sein d'une analyse qui inclut la superstructure socio-politique française. Nous devons donc nous demander si ce cas du Pays Basque est une anomalie, ou bien si ceci se retrouve dans la construction identitaire d'autres régions telles que la Bretagne (McDonald 1990, Sée 1977) ou la Corse (Bernabéu-Casanova 1997, Jaffe 1999). En d'autres mots, est-ce que l'exemple proposé ici relève de l'exception basque, ou est-il plutôt lié à une évolution plus globale dans les rapports entre régions et l'état? Nous avons pensé élucider cette problématique en suggérant que la construction de l'identité culturelle basque résulte d'une part des tensions exprimées entre différentes communautés basques, mais également des rapports asymétriques entre centre et périphérie qui se retrouvent dans de nombreux états.

Il est vrai que notre analyse relève principalement pour l'instant de documents rédigés par des représentants des institutions nationales et, moins souvent, locales. Mais cette interprétation n'est qu'une première étape dans notre investigation qui est encore en cours de réalisation. Nos recherches actuelles se concentrent sur le vingtième siècle et les expressions contemporaines de l'identité culturelle basque dans la vallée de Baïgorry. Nous voulons penser qu'une étude anthropologique des terres communales nous offrira un lien entre les situations historiques et contemporaines, entre la frontière et le centre, et ainsi pouvoir mieux percevoir les multiples et diverses influences sur la construction de l'identité culturelle au Pays Basque.

\section{Bibliographie :}

Anderson, B. (1983) Imagined Communities: Reflections on the Origins and Spread of Nationalism. New York: Verso.

Barth, F. (1969) Ethnic groups and boundaries: the social organization of cultural difference. Boston: Little Brown.

Bernabéu-Casanova, E. (1997) Le nationalisme Corse: genèse, succès et échec. Paris: L'Harmattan.

Brumfiel, E. : \& Fox, J. (1994) Factional competition and political development in the New World. New York: Cambridge University Press 
Cavaillès, H. (1910) Une fédération ancienne sous l'Ancien Régime.

Cavaillès, H. (1931a) La vie pastorale et agricole dans les Pyrénées. Paris: Armand Colin.

Cavaillès, H. (1931b) La transhumance pyrénéenne et la circulation dés troupeaux. Paris: Armand Colin.

Chaussier, J.D. (1989) Identités nationales et identités locales: le projet de création d'un département en Pays Basque. Thèse, Université de Bordeaux 2.

Cole, J. \& Wolf, E. (1974) The hidden frontier: ecology and ethnicity in an alpine valley. New York: Academic Press.

Donnan, H. \& Wilson, T. (1994) "An anthropology of frontiers" In H. Donnan \& T. Wilson (eds.) Border Approaches: anthropological perspectives on Frontiers, 1-14. New York: University Press of America.

Douglass, W. (1975) Echalar and Murelaga: Opportunity and Rural Depopulation in Two Spanish Basque Villages. New York: St. Martin's Press.

Douglass, W. (1977) “Borderland influences in a Navaresse village." In W. Douglass, Etulain, R. \& Jacobsen, W. (eds.) Anglo-American contributions to Basque studies : essays in honor of Jan Bilbao, 135-144. Reno, Nevada: Desert Research Institute.

Etchelecou, A. (1991) Transition démographique et système coutumier dans les Pyrénées Occidentales. Paris: Presses Universitaires de France.

García-Ruiz, J. \& Lasanta-Martínez, T. (1993) "Land-use conflicts as a result of landuse change in the central Spanish Pyrénées: a review." Mountain Research and Development 13 (3): 295-304.

Gauthier Dalché, P. (1990) “L'image des Pyrénées au Moyen Âge.” In P. Sénac (ed.) Frontières et espaces pyrénéens au Moyen Age, 15-37. Perpignan: Université de Perpignan.

Gellner, E. (1983) Nations and Nationalism. Ithaca, New York: Cornell University Press.

Gemie, S. (1998) "France and the Val d'Aran: Politics and Nationhood on the Pyrenean border, c. 1800-1825." European History Quarterly 28 (3): 311-345.

Gibson, B. \& Geselowitz, M. (1988) Tribe and Polity in Late Prehistoric Europe. New York: Plenum Press. 
Goyhenetche, M. (1975) L'oppression culturelle française en Pays Basque Nord. Donostia: Elkar.

Greenwood, D. (1976) Unrewarding wealth: the commercialization and collapse of agriculture in a Spanish Basque town. New York: Cambridge University Press.

Habermas, J. (1992) "Citizenship and National Identity: some reflections on the future of Europe." Praxis International 12 (1).

Jaffe, A. (1997) Ideologies in Action: Language Politics on Corsica. New York: Mouton de Gruyter.

Jenkins, B. (2000) "From Nation-Building to the Construction of Europe: the Lessons and Limitations of the French example." In Andrew, M. Crook \& M. Waller (eds.) Why Europe? Problems of Culture and Identity, vol 1. Basingstoke, UK: Macmillan Press.

Laborde, P. (1983) Le Pays Basque d'hier et d'aujourd'hui. Donostia: Elkar.

Lammers, B. (1999) "National Identity on the French Periphery: the end of Peasants into Frenchmen?" National Identities 1 (1):81-87.

Lefeb, re, T. (1933) Les modes de vie dans les Pyrénées Orientales. Paris: Armand Colin.

MacDonald, S. (1993) "Identity Complexes in Western Europe: Social Anthropological Perspectives." In S. MacDonald (ed.) Inside European Identities, 1-26. Oxford: Berg Publishers.

McDonald, M. (1990) "We are not French!": Language, Culture and Identity in Brittany. New York: Routledge Press.

Reid, D. (1985) The Miners of Decazeville: a Genealogy of Deindustrialization. Cambridge: Harvard University Press.

Renfrew, C. \& Cherry, J. (1986) Peer polity interaction and socio-political change. New York: Cambridge University Press.

Sahlins, P. (1989) Boundaries; the making of France and Spain in the Pyrénées. Berkeley: University of California Press.

Sée, H. (1977) Les Classes Rurales en Bretagne du xvte siècle à la Révolution. Brionne, France: Editions Gérard Monfort.

Viers, G. (1950) La vallée de Baïgorri, les paysages, la vie rurale. Thèse, Universitê de Bordeaux. 
Weber, E. (1983) La fin des terroirs: la modernisation de la France rurale: 18701914. Paris : Fayard.

Wintle, M. (1996) "Cultural Identity in Europe: shared experience" In M. Wintle Culture and Identity in Europe, 9-32. Brookfield, Vermont: Ashgate Publishing. 